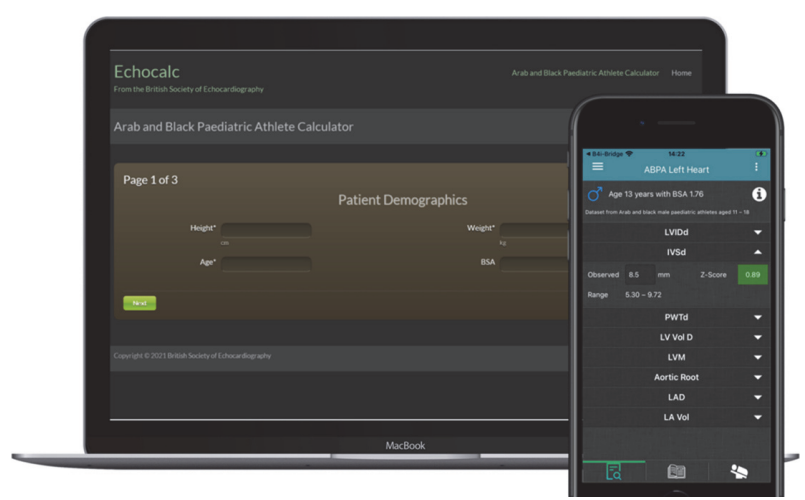

Abstract 156 Figure 2 Home page and pages for data entry of patient demographics and measured parameters allowing for automated calculation of Z-score online and on the EchoCalc app for use on android/iPhone. The data can be stored as a ".pdr document for emailing or storage on most browsers

Methods 417 (297 Arab, 120 black) male paediatric (11-18 years) athletes, were evaluated by $2 \mathrm{D}$ echocardiography as per the as per British Society of Echocardiography guidelines, and biological age (by radiological X-ray) assessment. Z scores involved allometric $\left(\mathrm{a}^{*}[\mathrm{BSA}]^{\wedge}\left(\left(\mathrm{b}+\mathrm{c}^{*}\right.\right.\right.$ chronological age $\left.)\right)$ modelling for measures of LV structure.

Results Residual linear regression, revealed normalised measures of LV to be independent of BSA, race, chronological and biological age. Z-scores for an athlete with a specific BSA and/or chronological age can be calculated 1) visually in the nomograms provided (figure 1) 2) automatically online/on the EchoCalc app (Figure 2), or 3) manually from by using the specified a, b, and c mean predicted parameters and $d$, e, and $f$ RSD for that parameter of LV structure from table $1: z=$ (obs- $\left(\mathrm{a}^{*}[\mathrm{BSA}]^{\wedge}\left(\left(\mathrm{b}+\mathrm{c}^{*}\right.\right.\right.$ chronological age $\left.\left.\left.)\right)\right)\right) /\left(\mathrm{d}+\left(\mathrm{e}^{*} \mathrm{BSA}\right)\right.$ $+\left(\mathrm{f}^{*}\right.$ chronological age $\left.)\right)$

Conclusion For the first time, we present BSA, race, chronological, and biological age independent nomograms and $\mathrm{Z}$ scores for LV, LA and Aortic size to BSA in male Arab and black paediatric athletes. Nomograms are provided to assist in the tracking of the paediatric athlete necessitating annual follow-up and online/app calculation to facilitate use in day-today practice (figures 1 and 2). In clinical context, we propose a new tool for differential diagnosis of physiological remodelling to an indicator of cardiac pathology in the cardiac screening of the paediatric athlete.

Conflict of Interest None

\section{18F-SODIUM FLUORIDE POSITRON EMISSION TOMOGRAPHY, AORTIC DISEASE ACTIVITY AND ISCHAEMIC STROKE RISK}

\footnotetext{
${ }^{1}$ Alexander Fletcher, ${ }^{1}$ Evangelos Tzolos, 'Shruti Joshi, ${ }^{2}$ Jacek Kwiecinski, ${ }^{1}$ Rong Bing, ${ }^{3}$ Maaz Syed, ${ }^{1}$ Mhairi Doris, ${ }^{1}$ Edwin van Beek, ${ }^{1}$ Alistiar Moss, ${ }^{4}$ Niki Walker, ${ }^{5}$ Nikhil Joshi, ${ }^{1}$ Tania Pawade, ${ }^{6}$ Philip Adamson, ${ }^{7}$ William Whiteley, ${ }^{7}$ Joanna Wardlaw, ${ }^{8}$ Piotr Slomka, ${ }^{1}$ Michelle Williams, ${ }^{1}$ David Newby, ${ }^{1}$ Marc Dweck. ${ }^{1}$ Centre for Cardiovascular Sciences, University of Edinburgh, Edinburgh, UK; ${ }^{2}$ Department of Interventional Cardiology and Angiology, Institute of Cardiology, Warsaw; ${ }^{3}$ BHF Department for Cardiovascular Sciences, University of Edinburgh; ${ }^{4}$ Golden Jubilee National Hosptial; ${ }^{5}$ Bristol Heart Institute, Bristol Royal Infirmary, University of Bristol; ${ }^{6}$ Christchurch Heart Institute, University of Otago, Christchurch; ${ }^{7}$ Centre for Clinical Brain Sciences, University of Edinburgh; ${ }^{8}$ Cedars-Sinai Medical Centre, Department of Imaging (Division of Nuclear Cardiology), Los Angeles, Un
}

10.1136/heartjnl-2021-BCS.154
Background Arterial 18F-sodium fluoride (18F-NaF) activity on positron emission tomography (PET) is a marker of active microcalcification and atherosclerosis. Coronary $18 \mathrm{~F}-\mathrm{NaF}$ activity (CMA) predicts coronary artery disease progression and subsequent myocardial infarction. We aimed to investigate whether aortic $18 \mathrm{~F}-\mathrm{NaF}$ activity (AMA) predicts thoracic aortic atherosclerotic disease progression and subsequent ischaemic stroke in patients with established cardiovascular disease.

Methods In a post-hoc observational cohort study, we evaluated AMA in patients with stable coronary artery disease $(n=239)$ or aortic stenosis $(n=158)$ who had underwent thoracic $18 \mathrm{~F}-\mathrm{NaF}$ PET and computed tomography (CT). We assessed the associations between AMA and progression of thoracic aortic calcification on follow up CT and subsequent ischaemic stroke or myocardial infarction.

Results In 141 patients with repeat CT imaging at $12.7 \pm 2.7$ months, AMA correlated with progression of thoracic aortic calcium scores $(r=0.21, p=0.011)$. In 397 patients, 16 had an ischaemic stroke and 25 had a myocardial infarction after 4.7 \pm 1.6 years. After adjusting for clinical risk factors, CMA and calcium scoring, AMA was associated with stroke (hazard ratio, 1.71 [95\% confidence interval 1.00-2.90], $\mathrm{p}=0.048]$ ). AMA was superior to clinical risk and calcium scores in identifying patients with stroke (c-statistic 0.76 versus 0.58 versus 0.63 respectively, $\mathrm{p}<0.05)$. Survival analysis demonstrated that AMA was associated with ischaemic stroke $(p<0.001)$ but not myocardial infarction $(p=0.45)$, whereas CMA was associated with myocardial infarction $(\mathrm{p}<0.001)$ but not stroke $(p=0.39)$.

Conclusions In patients with established cardiovascular disease, AMA is associated with progression of aortic atherosclerosis and future ischaemic stroke. Arterial $18 \mathrm{~F}-\mathrm{NaF}$ identifies localised areas of atherosclerotic disease activity that relate to regional atherothrombotic events.

Conflict of Interest none to delcare

\section{NON-INVASIVE NEAR-INFRARED FLOURESCENCE IMAGING OF MALODIALDEHYDE-OXIDISED LOW- DENSITY LIPOPROTEIN IN ATHEROSCLEROSIS}

Samata Pandey. Imperial College London, London, UK

\subsection{6/heartjnl-2021-BCS.155}

Introduction Atherosclerosis is an insidious disease where the accumulation of low-density lipoprotein in the arterial walls generates plaques, which may rupture leading to an untimely death or loss of quality of life. A key barrier for the treatment of atherosclerosis is identifying and predicting those plaques which are most vulnerable to rupture. This project aimed to bridge this diagnostic gap by using monoclonal antibodies for targeted imaging of malondialdehyde-low density lipoprotein (MDA-LDL), a component of vulnerable plaques.

Method A humanised monoclonal antibody (LO1-huFab) was expressed in human embryonic kidney cells (HEK)-293 cells with an unpaired sulfhydryl group for site-specific maleimide conjugation. The purified protein was site-specifically conjugated to a near-infrared fluorophore (LO1-huFab-750) and one nanomole injected in LDL receptor-deficient (LDLR-/-) and wild-type (C57/BL6) mouse model. Mice were additionally co-injected with fluorophores targeting matrix metalloproteinases (MMPSense) and integrin a5b3 (IntegriSense). Non-invasive fluorescence molecular tomography imaging was 